\title{
Numerical simulation of turbulent flow physic in a tube array
}

\author{
Y. Hoarau and M. Braza and G. Harran and E. Longatte and F. Baj and T. Marcel
}

\begin{abstract}
The prediction of fluid-elastic instabilities developing in a tube bundle is of major importance in the design of modern heat exchangers for nuclear reactor cooling to prevent accidents associated with fluid-elastic instabilities leading to flutter, material fatigue, shocks between beams and damage of the solid walls. The fluid-elastic instabilities in tube arrays appear in the laminar regime and persist in the high Reynolds number turbulent flow. They are governed by non-linear interaction between fluid-elastic instability and turbulence. It is of major importance for the design to accurately predict the unsteady loads under turbulent flow and the amplification of the fluid-elastic instabilities. This work focuses on the numerical simulation of turbulent flow in tube bundle and prediction of fluid-elastic instabilities.
\end{abstract}

\section{Introduction}

There is a considerable effort in the state-of-the-art to improve methodologies able to capture the fluid-structure interaction under turbulent flow, and to assess critical thresholds. These aspects are challenging for the research applications involving the nuclear power industry. The present work is part of the BARESAFE project funded by the French National Research Agency (ANR) with LAMSID,

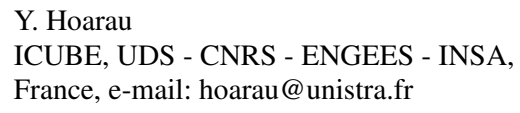

M. Braza and G. Harran and T. Marcel

Institut de Mécanique des Fluides de Toulouse, UMR CNRS 5502, France

E. Longatte

Laboratoire de Mécanique des Structures Industrielles Durables, UMR EDF CNRS 2832, France

F. Baj

Commissariat à l’Energie Atomique CEA / Saclay, France 
EDF, CEA, IMFT, ICUBE, IRIT and AREVA. The BARESAFE project (Numerical modeling of multi-scale fluid solid coupled systems : advanced hybrid methods for reduction of uncertainty on stability limits and optimization of nuclear safety barrier reliability) is a research project involved in the development of modeling and advanced numerical methods for simulation of large size systems involving multi-physics in the field of mechanics. It addresses the hard issue of stability analysis of dynamical systems submitted to external turbulent flows and aims to establish accurate stability maps applicable for heat exchanger design. The purpose is to provide stability limit dimensionless modeling suitable in a variety of configurations with a maximal accuracy in spite of the large scale of the systems to be considered. The challenge lies in predicting local effects possibly impacting global systems. Therefore the combination of several strategies convenient simultaneously for multi-physics, multi-scale and large size system computation is required. Based on empirical concepts, the heuristic models currently used in the framework of standard stability analysis suffer from a lack of predictive capabilities [Blevins(1974), Blevins(1979), Tanaka and Takahara(1981), Tanaka and Takahara(1982), Chen(1983), Paidoussis and Price(1988)],

[Lever and Weaver(1982)]. On the other side, numerical approaches based on fullycoupled fluid solid dynamics system computation remain expensive due to the multiphysics patterns of physics and the large number of degrees of freedom to be involved. In this context, since experimentation is not reachable and numerical simulation is unavoidable but prohibitive, the project proposes an hybrid strategy in order to take advantages from both numerical local and empiric global solutions.

The present study focuses on the improvement of the numerical simulation of the fluid-structure interaction in the cylinders arrays, especially in the parametric range favoring the amplification and negative damping of flutter instability. The main objective is to analyze the physics of the appearance of Movement Induced Vibrations in connection with the instabilities coming from the fluid part in an array bundle. Concerning turbulence modeling methods, among URANS approaches, the Organised Eddy Simulation [Braza et al.(2006), Bourguet et al.(2008)], OES has been used to capture the coherent flow patterns and non-equilibrium effects associated with the fluid-elastic instabilities. Furthermore, the OES version of the Delayed Detached Eddy Simulation and the Scale Adaptive Simulation has been used for the 3-D simulations.

First we focused on a two-dimensional study in static configuration, in order to assess the predictive ability of 2-D mechanisms. Secondly, the spontaneous motion of a central cylinder, in SDOF (single degree of freedom) vertical displacement is studied, below and beyond critical values for the appearance of flutter instability in the array of cylinders. Then the three-dimensional flow structure is studied by OES-DDES [Skopek et al.(2012)] and SAS [Menter and Egorov(2005), Menter and Bender(2003)].

Only the static approach is presented in this paper. 


\section{Problem set-up}

The configuration fit an experimental set-up (DIVA) designed by the CEA where 20 cylinders are arranged in 5 columns with a pitch of $1.5 D, D$ being the diameter of the cylinders. The Reynolds number is 20.000 which corresponds to an intertube Reynolds number of 60.000. The grid (Fig. 1) is composed with 392600 cells distributed on 80 structured blocs. On the entry and on the outlet boundary, the conditions are :

- inlet : far field boundary condition using characteristic variables,

- outlet : subsonic outflow with imposed static pressure based on Riemann invariants.

- top-bottom : no-slip walls.


Fig. 1 Grid overview

\section{Numerical modeling}

The simulations are performed with our parallelized Navier-Stokes Multi-Block $(N S M B)$ solver [Vos et al.(1998)]. NSMB solves the compressible Navier-Stokes 
equations using a finite volume formulation on Multi-Block structured grids. Various spatial discretization schemes are available like Jameson's central difference, Roe or AUSM+. The time integration is based on the full matrix implicit LU-SGS (Lower-Upper Symmetric Gauss-Seidel) method [Yoon and Jameson(1986)] and on the dual-time stepping. In the present work, the artificial compressibility method [Chorin(1968)] is employed. NSMB is parallelized using the Message Passing Interface.

\section{Turbulence modeling}

The URANS turbulence models used in this study are the [Spalart and Allmaras(1992)], the $k-\omega$ models (baseline and SST) of [Menter(1994)], the OES $k-\varepsilon$ and the OES $k-\omega$ baseline models, described in [Braza et al.(2006), Bourguet et al.(2008)]. The Delayed Detached Eddy Simulation, DDES is employed by using for the RANS part the turbulence length scale from the OES method.

The association of the OES modeling in the RANS part of the DES/DDES is reported in a number of studies of the present research group, that have been the object of applications in a variety of test cases in the European research programs DESIDER (Detached Eddy Simulation for Industrial Aerodynamics) and ATAAC (Advanced Turbulence Modeling for Aeronautics Applications Challenges) in aeronautics. The introduction of a turbulence length scale from OES in the Delayed DES, DDES [Spalart et al.(2006)] is reported in [Haase and Revell(2009)], [Bourguet et al.(2008)] and in [Skopek et al.(2012)]. The use of this variety of turbulence models allows assessment of their predictive capabilities in respect of the strongly detached unsteady flow character in the array of cylinders.

The OES modeling approach is used to capture the two aspects of fluid structure instability : organized and chaotic.

Fig. 2 Dual spectrum splitting : the distinction between the structures to be resolved and those to be modeled is based upon their organized or random character. Part (2) of the non-equilibrium energy spectrum has to be modeled by reconsidering advanced statistical turbulence modeling efficient in high-Re wall flows, due to the inertial-range modulation from equilibrium turbulence, schematically shown on the right.

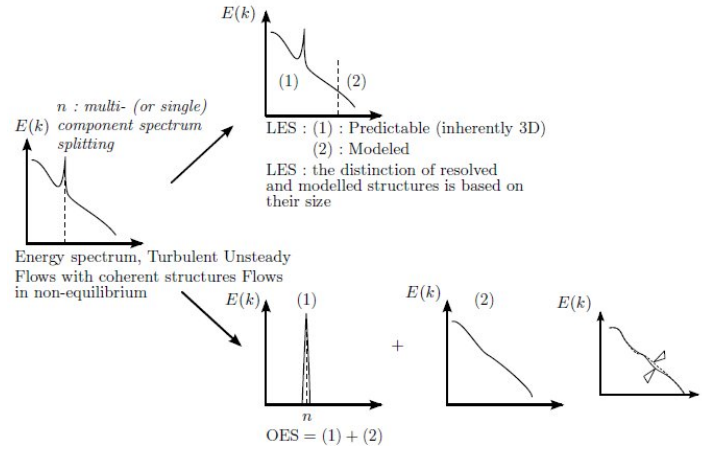


In this approach, the turbulent spectrum is decomposed in a first part regrouping all the coherent structures (resolved part) and in a second part regrouping all the chaotic processes independently on their size (spectrum to be modeled), as presented schematically in Fig. 2. It is recalled that in LES the distinction is done according to the size of the structures which limits its application to moderate Reynolds numbers. The fact that part of the spectrum to be modeled in OES extend from the low to the high frequencies allows the use of statistical turbulence modeling with appropriate modification due the non-equilibrium. In the time-domain, the equations are the phase-averaged NavierStokes equations. Due to the nonlinear interaction between the coherent part and the non-coherent one, there is a slope modification of the inertial part in the spectrum, in the vicinity of the peak. This yields to a reconsideration of the eddy-diffusion coefficient for the class of the two-equation modeling, as well as improved damping functions to attenuate turbulence towards the wall.

\section{Results}

The unsteady loads obtained with this four turbulence models are examined and their average values, their minimum and maximum values (Table 1) their frequency distribution (Table 2) are compared.

\begin{tabular}{|c|c|c|c|}
\hline Models & $C_{L, R M S}$ & $C_{L, \min .}$ & $C_{L, \max .}$ \\
\hline \hline Spalart-Allmaras & 0.44 & -0.89 & 1.04 \\
$k-\omega-S S T$ & 0.19 & -0.68 & 0.58 \\
$k-\omega-\mathrm{BL}-\mathrm{OES}$ & 0.65 & -1.84 & 1.69 \\
$k-\varepsilon-$ OES & 0.46 & -1.08 & 1.11 \\
\hline DIVA Experiment & 0.45 & -1.86 & 2.33 \\
\hline
\end{tabular}

Table 1 Lift coefficient of unsteady loads

We have a good match between the turbulent simulations and the experimental data. The $k-\varepsilon-$ OES and Spalart-Allmaras approaches give the best prediction of the RMS values of the lift coefficient. However, for the extrema values, the $k-$ $\omega-\mathrm{BL}-\mathrm{OES}$ prediction brings the best results.

Extrema values of the lift coefficient are also important in a study on the fluidstructure interaction. Indeed, it is possible that not only the signal strength (RMS values) but also the extent of the values will trigger the instability. This is why the prediction of unsteady loads have to be accurate for these two aspects. In this context, OES approaches seem to bring better results than conventional URANS approaches.

This can be explained by the fact that this complex flow configuration of the tube bundle at this Reynolds number caused a mixed boundary layer. OES approach allow to have a better capture of the mixed boundary layer than the classical URANS approaches. 
Concerning the frequencies, results are presented on Power Spectral Density (PSD) on Fig. 3.


Fig. 3 Power spectrum density of the lift coefficient for the models SA, $k-\omega-\mathrm{SST}, k-$ $\omega$-Baseline-OES and $k-\varepsilon$-OES closures with hanning windows

We can find also a good match between models and experimental data. This is the first time to our knowledge that unsteady numerical simulations have so good agreement with this test bench of the configuration of a tube bundle under cross flow at a Reynolds number of 20.000.

OES approaches seem to predict a more accurate power level than the URANS classical ones which slightly underestimate this level.

Three predominant frequencies appear on the spectra. After a study of the cartography of the separation point, we have linked the values of these frequencies to known physical phenomena : the Von Kármán vortex shedding $S t_{V K}$, the confinement phenomenon $S t_{C}$ and the shear layer phenomenon $S t_{S L}$.

We can define a Strouhal number with the free stream velocity, but also a Strouhal number with inter-tube velocity, which we denote $S t$, , it

$$
S t_{\cdot, i t}=f d u_{i t}=\left(P^{*}-1 P^{*}\right) S t \quad \text { with } \quad P^{*}=P d
$$

with $P^{*}$ the reduced step. Values of predominant frequencies are summarized in Tab. 2.

The inter-tube value observed for the Von Kármán Strouhal number is nearly that the conventional value known for a Reynolds number of 60.000 for one cylinder $\left(S t_{c y l} \approx 0.2\right)$. Furthermore the four models predict almost the same values for the three predominant frequencies.

As for the study of the cartography of the separation point, we focused only on the central cylinder and we looked at the position of the point of detachment on the wall of the cylinder for a series of snapshots. We found a periodical appearance of 


\begin{tabular}{|c||c|c||c|c|}
\hline Models & $S t_{V K}$ & $S t_{V K, i t}$ & $S t_{C}$ & $S t_{S L}$ \\
\hline Spalart-Allmaras & 0.34 & 0.11 & 0.99 & 2.25 \\
$k-\omega-$ SST & 0.36 & 0.12 & 0.99 & 2.25 \\
$k-\omega-$ OES & 0.39 & 0.13 & 1.01 & 2.25 \\
$k-\varepsilon-$ OES & 0.39 & 0.13 & 1.01 & 2.25 \\
\hline
\end{tabular}

Table 2 Values of predominant frequencies

grounds which correspond to the predominant frequencies of the spectral study. The frequency which is linked to the Von Kármán vortex shedding phenomenon, whose value is rather low (relative to the other two), is located to the rear of the cylinder. One that matches the shear layer phenomenon is located just above this area with a higher value. Finally, the third frequency is linked to a phenomenon related to the impact of coherent structures on the zone $|\alpha| \approx 60^{\circ}$. This zone corresponds to the area where the structures issued from the previous rows of cylinders are crashing. That's why this predominant frequency is linked to a confinement phenomenon, caused by the geometry of the tube bundle.

\section{Conclusions}

The present study shows the prediction of unsteady loads in a tube bundle at high Reynolds number in the context of fluid-structure interaction.

URANS and OES turbulence modeling comparison on the static configuration is performed. The oscillatory characteristics is well predict, particularly concerning the power spectrum density. OES modeling improves the coherent structure prediction in the cylinder wake, which is under-predicted with the classical URANS approach.

There is a good match with the experimental data, even if the simulation have been computed in two dimensions. The 3-D simulations are in progress in order to find the purely 3-D phenomenon.

There are three phenomena which involve three predominant frequencies in the tube bundle under cross flow at Reynolds number of 20.000: the Von Kármán vortex shedding, the confinement and the shear layer. The demonstration of the existence of this three predominant frequencies is a first step to the numerical study of fluidelastic instability.

The classical URANS and OES approaches are good tools to compute simulations at this Reynolds number. DNS is too expensive in term of computational cost. So there is an obvious interest to use hybrid approaches for 3-D simulation.

Acknowledgements This study was made using high performance calcul possibility of nationals centers as CINES, TGCC/CCRT and the computing center of the Strasbourg University. This work was conducted in the context of the BARESAFE project (http://www-imfs.ustrasbg.fr/baresafe/index.html), ANR 11-MONU-004. 


\section{References}

[Blevins(1974)] Blevins, R., 1974. Fluid-elastic whirling of a tube row. J. of Pressure Vessel Tech. 96, 263-267.

[Blevins(1979)] Blevins, R. D., 1979. Formulas for natural frequency and mode shapes. Krieger Publishing Company.

[Bourguet et al.(2008)] Bourguet, R., Braza, M., Harran, G., El Akoury, R., 2008. Anisotropic organised eddy simulation for the prediction of non-equilibrium turbulent flows around bodies. J. of Fluid and Struct. 24(8), 1240-1251.

[Braza et al.(2006)] Braza, M., Perrin, R., Hoarau, Y., 2006. Turbulence properties in the cylinder wake at high reynolds number. J. of Fluid and Struct. 22, 7551-771.

[Chen(1983)] Chen, S. S., 1983. Instability mechanisms and stability criteria of a group of circular cylinders subjected to cross flow. part i: Theory. J. of Vibration, Acoustics, Stress and Reliability in Design 105(1), 51-58.

[Chorin(1968)] Chorin, A., 1968. Numerical solution of the navier-stokes equations. J. Math. Computation 22, 745.

[Haase and Revell(2009)] W. Haase, M. Braza, M., Revell, A., 2009. DESider A European Effort on Hybrid RANS-LES Modelling. Vol. 103 of Notes on Numerical Fluid Mechanics and Multidisciplinary Design. Springer.

[Lever and Weaver(1982)] Lever, J., Weaver, D., 1982. A theoretical model for fluid-elastic instability in heat exchanger tube bundles. J. of Pressure Vessel Tech. 104, 147-158.

[Menter(1994)] Menter, F., 1994. Two-equation eddy-viscosity turbulence models for engineering applications. AIAA J. 32(8), 1598-1605.

[Menter and Bender(2003)] F.R. Menter, M. K., Bender, R., 2003. A scale-adaptive simulation model for turbulent flow prediction. AIAA paper 2003-0767, Reno, NV.

[Menter and Egorov(2005)] Menter, F., Egorov, Y., 2005. A scale-adaptive simulation model using two-equation models. AIAA paper 2005-1095, Reno, NV.

[Paidoussis and Price(1988)] Paidoussis, M. P., Price, S. J., 1988. The mechanisms underlying flow-induced instabilities of cylinder arrays in crossflow. J. of Fluid Mech. 187, 45-59.

[Schwamborn and Strelets(2012)] Schwamborn, D., Strelets, M., 2012. Ataac an eu-project dedicated to hybrid rans/les methods. Notes on Num. Fluid Mech. and Multidisciplinary Design $117,59-75$.

[Skopek et al.(2012)] Skopek, M. G., Braza, M., Hoarau, Y., Thiele, F., 2012. Hybrid rans-les modeling of a strongly detached turbulent flow around a tandem cylinders configuration. In: S. Fu, W. Haase, S. P., Schwamborn, D. (Eds.), Progress in Hybrid RANS-LES Modelling, Papers Contributed to the 4th Symposium on Hybrid RANS-LES Methods, Beijing, China, September 2011. Notes on Num. Fluid Mech. and Multidisciplinary Design, Vol. 117. Springer-Verlag, Berlin, pp. 219-229.

[Spalart and Allmaras(1992)] Spalart, P., Allmaras, S., 1992. A one-equation turbulence model for aerodynamic flows. AIAA Paper 439.

[Spalart et al.(2006)] Spalart, P., Deck, S., Shur, M., Squires, K., Strelets, M., Travin, A., 2006. A new version of detached-eddy simulation, resistant to ambiguous grid densities. Theor. Comput. Fluid Dyn. 20, 181-195.

[Tanaka and Takahara(1981)] Tanaka, H., Takahara, S., 1981. Fluid elastic vibration of tube array in cross flow. Journal of Vound and Vibration 77, 19-37.

[Tanaka and Takahara(1982)] Tanaka, H., Takahara, S., 1982. Flow induced vibration of tube arrays with various pitch-to-diameter ratios. J. Pressure Vessel Technol. 104(3), 168-174.

[Yoon and Jameson(1986)] Yoon, S., Jameson, A, 1986. A Multigrid LU-SSOR Scheme for Approximate Newton Iteration Applied to the Euler Equations. NASA-CR-179524, 1986

[Vos et al.(1998)] Vos, J., Chaput, E., Arlinger, B., Rizzi, A., Corjon, A., 1998. Recent advances in aerodynamics inside the NSMB (Navier-Stokes Multi-Block) consortium. AIAA Paper 0802. 